\title{
Comment on "Critical behavior of the chain-generating function of self-avoiding walks on the Sierpinski gasket family: The Euclidean limit"
}

\author{
Sava Milošević \\ Faculty of Physics, University of Belgrade, P.O.Box 368, \\ 11001 Belgrade, Serbia, Yugoslavia \\ Ivan Živić \\ Faculty of Natural Sciences and Mathematics, University of Kragujevac, \\ 34000 Kragujevac, Serbia, Yugoslavia \\ Sunčica Elezović-Hadžić \\ Faculty of Physics, University of Belgrade, P.O.Box 368, \\ 11001 Belgrade, Serbia, Yugoslavia
}

(March 14, 2018)

\begin{abstract}
We refute the claims made by Riera and Chalub [Phys. Rev. E 58, 4001 (1998)] by demonstrating that they have not provided enough data (requisite in their series expansion method) to draw reliable conclusions about criticality of self-avoiding walks on the Sierpinski gasket family of fractals.

05.40.+j, 05.50+q, 64.60.Ak, 61.41.+e
\end{abstract}

Typeset using REVTEX 
The self-avoiding walk (SAW) on a lattice is a random walk that must not contain selfintersections. The criticality of SAWs has been extensively studied as a challenging problem in statistical physics on the Euclidean lattices and on fractal lattices as well. Accordingly, the question has been posed whether the critical behavior of SAWs on a Euclidean lattice can be retrieved via a limit of infinite number of fractals whose properties gradually acquire the corresponding Euclidean values. In this Comment we scrutinize the methods used so far to answer the foregoing question.

The most frequently studied infinite family of finitely ramified fractals appears to be the Sierpinski gasket (SG) family. Each member of the SG family is labeled by an integer $b$ $(2 \leq b \leq \infty)$, and when $b \rightarrow \infty$ both the fractal $d_{f}$ and spectral $d_{s}$ dimension approach the Euclidean value 2. Concerning the study of the criticality of SAWs, these fractal lattices are perfect objects for application of the renormalization group (RG) method, due to their intrinsic dilation symmetry (the so-called self-similarity) and their finite ramification. The latter property enables one to construct a finite set of the RG transformations and therefrom an exact treatment of the problem. This treatment was first applied by Dhar [1], for $b=2$, and later it was extended [2] up to $b=8$. The obtained results of the corresponding critical exponents, for the finite sequence $2 \leq b \leq 8$, were not sufficient to infer their relation to the relevant Euclidean values. However, these results inspired the finite-size scaling (FSS) approach to the problem [3], which brought about the prediction that the SAW critical exponents on fractals do not necessarily approach their Euclidean values when $b \rightarrow \infty$. Indeed, Dhar [3] found that the critical exponent $\nu$, associated with the SAW end-to-end distance, tends to the Euclidean value 3/4, whereas the critical exponent $\gamma$, associated with the total number of distinct SAWs, approaches 133/32, being always larger than the Euclidean value $\gamma_{E}=43 / 32$.

The intriguing FSS results motivated endeavors to extend the exact RG results beyond $b=8$. However, since this extension appeared to be an arduous task, a new insight was needed. This insight came from a formulation of the Monte Carlo renormalization group (MCRG) method for fractals [4,5], which produced values for $\nu$ and $\gamma$ up to $b=80$. For $2 \leq b \leq 8$ the MCRG findings deviated, from the exact values, at most $0.03 \%$, in the case of $\nu$, and $0.2 \%$ in the case of $\gamma$. In addition, the behavior of the entire sequence of the MCRG findings, as a function of $b$, supported the FSS predictions.

Recently, Riera and Chalub [6] made a different type of endeavor to obtain results for the critical exponent $\gamma$ for large $b$, by applying an original series expansion method [7]. However, the data of Riera and Chalub (RC) display a quite different behavior than the MCRG results (see Fig. 1). As regards comparison of the $\mathrm{RC}$ results with the available exact RG results [2], one may notice a surprising discrepancy: for $b=7$ the $\mathrm{RC}$ result deviates $19 \%$ (which should be compared with the respective MCRG deviation $0.13 \%$ ), while for $b=8$ the RC result deviates $33 \%$ from the exact result (which is again much larger than the corresponding MCRG deviation $0.15 \%$ ). On the other hand, concerning behavior of $\gamma$ beyond $b=8$, the RC results start to decrease, whereas the MCRG results monotonically increase. Furthermore, Riera and Chalub [6] claimed that, in contrast with the FSS prediction [3], $\gamma$ should approach the Euclidean value $43 / 32=1.34375$, in the limit of very large $b$. These discrepancies call for inspection of both methods, that is, of the MCRG technique $[4,5]$ and the series expansion 
method [6,7]. We are going first to reexamine our MCRG approach, and then we shall comment on the applicability of the RC series expansion method for large $b$.

We have found that the best way to check the validity of the MCRG method, for large $b$, is to apply it in a case of a random walk model that is exactly solvable for all possible $b$. To this end, the so-called piecewise directed walk (PDW) $[8,9]$ turned out to be quite appropriate. The PDW model describes such a random self-avoiding walk on SG fractals in which the walker is allowed to choose randomly, but self-similarly, limited number of possible step directions [8]. This model corresponds to the directed random walk on Euclidean lattices, in which case $\nu_{E}=1$ and $\gamma_{E}=1$. By applying the exact $\mathrm{RG}$ approach the critical exponent $\nu$ and $\gamma$ for PDW have been obtained [8,9] for each $b(2 \leq b<\infty)$. Moreover, it was demonstrated exactly that $\nu$ approaches the Euclidean value $\nu_{E}=1$, while $\gamma$ tends to nonEuclidean value $\gamma=2$, when $b \rightarrow \infty$. Here, we apply the MCRG method (used in the case of SAW in [4,5]) to calculate $\nu$ and $\gamma$ of the PDW model for $2 \leq b \leq 100$. Our results, together with the exact findings, are presented in Table I and depicted in Fig. 2 and Fig. 3. One can see that, in the entire region under study, the agreement between the MCRG results and the exact data is excellent. Indeed, the deviation of the MCRG results for $\nu$ from the corresponding exact results is at most $0.08 \%$, while in the case of $\gamma$ it is at most $0.8 \%$. This test of the MCRG method provides novel reliability for its application in studies of random walks on SG with large $b$.

Because of the confirmed reliability of the MCRG method, and because Riera and Chalub [6] have obtained quite different results, in the case of SAWs on SG, we have reason to assume that their conclusions were obtained in a wrong way. Thus, we may pose a question what was wrong in the application of the series expansion method in the work of Riera and Chalub [6]. Let us start with mentioning that in the series expansion study of SAW the first task is to determine the number $c_{n}(b)$ of all possible SAWs for a given number $n$ of steps, where $1 \leq n \leq n_{\max }$. Of course, in practice, it is desirable to perform this enumeration for very large $n_{\max }$, as the corresponding numbers $c_{n}(b)$ of all $n$-step SAWs represent coefficients of the relevant generating function $C_{b}(x)=\sum_{n=1}^{\infty} c_{n}(b) x^{n}$ (where $x$ is the weight factor for each step), whose singular behavior determines critical exponents of SAWs. In order to take into account existence of the SG lacunarity, the average end-to-end distance of the set of $n$-step SAWs should be larger than the size of the smallest homogeneous part of the SG fractal [3], that is, $n_{\max }$ should be larger than $b^{4 / 3}$. In a case when the number of steps is smaller than $b^{4 / 3}$ the corresponding SAWs percieve the underlying fractal lattice as a Euclidean substrate. In order to make it more transpicuous, we present in Fig. 4 the curve $n=b^{4 / 3}$ which divides the $(b, n)$ plane in two regions so that one of them corresponds to the fractal behavior of $n$-step SAWs, while the other corresponds to the Euclidean behavior. In the same figure, for a given $b$, we also depict the number of coefficients (empty small triangles) that were obtained by Riera and Chalub [6] for the corresponding SG fractal, in their series expansion approach. One can see that only for $2 \leq b \leq 8$ Riera and Chalub generated sufficient number of coefficients $c_{n}(b)$ so as to probe the fractal-behavior region (in which the condition $n_{\max } \geq b^{4 / 3}$ is satisfied). On the other hand, for $b>8$, in all cases studied, the maximum length $n_{\max }$ of enumerated SAWs [6] is not larger than 16, and thereby the corresponding generating functions $C_{b}(x)$ remain in the domain of the Euclidean behavior (see Fig. 4). For instance, in order to study criticality of SAWs on the SG fractal, for $b=80$, it is prerequisite to calculate all coefficients $c_{n}(b)$ in the interval $1 \leq n \leq n_{\max }$, where $n_{\max }$ 
must be larger than $80^{4 / 3} \approx 339$, which is far beyond $n_{\max }=13$ that was reached in [6] for $b=80$. This explains why the RC results for the SG critical exponent $\gamma$ (see Fig. 1), with increasing $b$, wrongly become closer to the Euclidean value 1.34375.

The problems discussed above, that is, the problems with not long enough SAWs, do not appear in the MCRG study of SAWs on the SG fractals, as the RG method in general takes into account SAWs of all length scales. Incidentally, we would like to mention that in [6] it was erroneously quoted that in the MCRG studies [4,5] one Monte Carlo (MC) realization corresponds to simulation of one SAW. In fact, one MC realization implies simulations of all possible walks on the fractal generator, which appears to be the smallest homogeneous part of the SG fractal. For instance, for the $b=80 \mathrm{SG}$ fractal, in order to calculate the critical exponent $\gamma$, in one MC realization we simulated [5] all $n$-step SAWs with $n$ ranging between 1 and 3240.

Finally, we would like to comment on the analytical argument, given in [6], which was assumed to support the claim $\lim _{b \rightarrow \infty} \gamma=\gamma_{E}$. One can observe that the corresponding argument does not exploit particular properties of the SAWs studied. Thus, if the argument were valid, it could be applied to other types of SAWs on fractals leading to the same conclusion $\lim _{b \rightarrow \infty} \gamma=\gamma_{E}$. However, the case of the PDW discussed in this comment is a definite counterexample to the foregoing conclusion, as it was rigorously demonstrated $[8,9]$ that in this case $\lim _{b \rightarrow \infty} \gamma \neq \gamma_{E}$.

In the conclusion, let us state that in this comment we have vindicated that the MCRG technique for studying the SAW critical exponents on fractals is a reliable method and a valuable tool in discussing the query whether the critical behavior of SAWs on a Euclidean lattice can be achieved through a limit of infinite number of fractals whose properties gradually acquire the corresponding Euclidean values. On the other hand, we have demonstrated that Riera and Chalub [6], in an attempt to answer the mentioned query by applying the series expansion method, have not provided sufficient number of numerical data for a study of criticality of SAWs on the SG fractals with finite scaling parameters $b$. Therefore, any inference from such a set of data about the large $b$ behaviour of the critical exponent $\gamma$ cannot be tenable.

We would like to acknowledge helpful and inspiring correspondence with D. Dhar concerning the matter discussed in this Comment. 


\section{REFERENCES}

[1] D. Dhar, J. Math. Phys. 19, 5 (1978).

[2] S. Elezović, M. Knežević and S. Milošević, J. Phys. A 20, 1215 (1987).

[3] D. Dhar, J. Physique 49, 397 (1988).

[4] S. Milošević and I. Živić, J. Phys. A 24, L833 (1991).

[5] I. Živić and S. Milošević, J. Phys. A 26, 3393 (1993).

[6] R. Riera and F. A. C. C. Chalub, Phys. Rev. E 58, 4001 (1998).

[7] F. A. C. C. Chalub, F. D. A. Aarão Reis, and R. Riera, J. Phys. A 30, 4151 (1997).

[8] S. Elezović-Hadžić, S. Milošević, H. W. Capel, and G. L. Wiersma, Physica A 149, 402 (1988).

[9] S. Elezović-Hadžić, S. Milošević, H. W. Capel, and Th. Post, Physica A 177, 39 (1991). 


\section{FIGURES}

FIG. 1. The SAW critical exponent $\gamma$ as a function of $1 / b$. The solid triangles represent results of the MCRG calculation [5], while the open triangles represent results obtained by Riera and Chalub (RC) via the series expansion method [6]. In both cases the solid lines that connect the data symbols serve as the guide to the eye. One should observe unusually large error bars in the case of RC results, whereas in the case of the MCRG results the error bars lie within the data symbols. The horizontal dashed line represents the Euclidean value $\gamma_{E}=43 / 32$ (which is also indicated by the solid horizontal arrow).

FIG. 2. The PDW critical exponent $\nu$ as a function of $1 / b$. The solid line represents exact results [8], while the solid triangles represent the MCRG results (see Table I). The error bars for the MCRG results lie within the data symbols. The inset is given in order to depict the limiting value of $\nu$ (solid circle) which coincides with Euclidean value $\nu_{E}=1$.

FIG. 3. The PDW critical exponent $\gamma$ as a function of $1 / b$. The solid line represents exact results [9], while the solid triangles represent the MCRG results (see Table I). The data error bars for the MCRG results for $b$ up to 30 are invisible, while for larger $b$ the error bars are comparable with the size of the symbols. The inset is given in order to depict the limiting value $\gamma=2$ (solid circle) which is different from the Euclidean value $\gamma_{E}=1$.

FIG. 4. The schematic representation of the fractal and Euclidean behavior of SAWs on the SG fractals. Regions of the two different behaviors are separated by the solid line $n=b^{4 / 3}$. The height of each vertical line (comprised of small triangles) corresponds to the maximum length of the $n$-step SAWs enumerated in the series expansion approach by Riera and Chalub [6], for a given $b$. In order to study criticality of SAWs on the SG fractals it is necessary to extend the vertical lines beyond the solid curve. Accordingly, one should observe that results of Riera and Chalub [6] do not probe the fractal region for $b>8$. 


\section{TABLES}

TABLE I. The exact RG and MCRG (in the brackets) results of the PDW critical exponents $\nu$ and $\gamma$ for the SG fractals for $2 \leq b \leq 100$. The exact RG results have been obtained in [8,9], while the MCRG results are calculated in the present work.

\begin{tabular}{ccc}
\hline \hline$b$ & $\nu$ exact(MC) & $\gamma$ exact(MC) \\
\hline 2 & $0.79870(0.79801 \pm 0.00075)$ & $0.9520(0.9503 \pm 0.0018)$ \\
3 & $0.82625(0.82683 \pm 0.00043)$ & $0.9631(0.9638 \pm 0.0014)$ \\
4 & $0.84311(0.84332 \pm 0.00010)$ & $0.9673(0.9665 \pm 0.0011)$ \\
5 & $0.85469(0.85470 \pm 0.00008)$ & $0.9695(0.9690 \pm 0.0011)$ \\
6 & $0.86329(0.86329 \pm 0.00006)$ & $0.9711(0.9707 \pm 0.0012)$ \\
7 & $0.87000(0.86992 \pm 0.00006)$ & $0.9726(0.9723 \pm 0.0012)$ \\
8 & $0.87542(0.87536 \pm 0.00005)$ & $0.9742(0.9732 \pm 0.0012)$ \\
9 & $0.87992(0.87978 \pm 0.00004)$ & $0.9759(0.9749 \pm 0.0013)$ \\
10 & $0.88374(0.88370 \pm 0.00013)$ & $0.9777(0.9770 \pm 0.0014)$ \\
12 & $0.88992(0.88984 \pm 0.00011)$ & $0.9815(0.9812 \pm 0.0015)$ \\
15 & $0.89679(0.89662 \pm 0.00009)$ & $0.9877(0.9839 \pm 0.0016)$ \\
17 & $0.90035(0.90040 \pm 0.00008)$ & $0.9919(0.9911 \pm 0.0016)$ \\
20 & $0.90467(0.90460 \pm 0.00007)$ & $0.9982(0.9946 \pm 0.0018)$ \\
25 & $0.91011(0.91002 \pm 0.00006)$ & $1.0084(1.0005 \pm 0.0019)$ \\
30 & $0.91417(0.91415 \pm 0.00002)$ & $1.0180(1.0181 \pm 0.0020)$ \\
35 & $0.91736(0.91727 \pm 0.00005)$ & $1.0270(1.0194 \pm 0.0022)$ \\
40 & $0.91996(0.91995 \pm 0.00005)$ & $1.0354(1.0368 \pm 0.0023)$ \\
50 & $0.92399(0.92393 \pm 0.00004)$ & $1.0504(1.0457 \pm 0.0025)$ \\
60 & $0.92701(0.92704 \pm 0.00003)$ & $1.0634(1.0696 \pm 0.0026)$ \\
70 & $0.92940(0.92934 \pm 0.00003)$ & $1.0750(1.0789 \pm 0.0028)$ \\
80 & $0.93136(0.93136 \pm 0.00001)$ & $1.0853(1.0854 \pm 0.0029)$ \\
90 & $0.93300(0.93300 \pm 0.00001)$ & $1.0945(1.0948 \pm 0.0031)$ \\
100 & $0.93441(0.93437 \pm 0.00002)$ & $1.1029(1.1089 \pm 0.0032)$ \\
\hline \hline
\end{tabular}




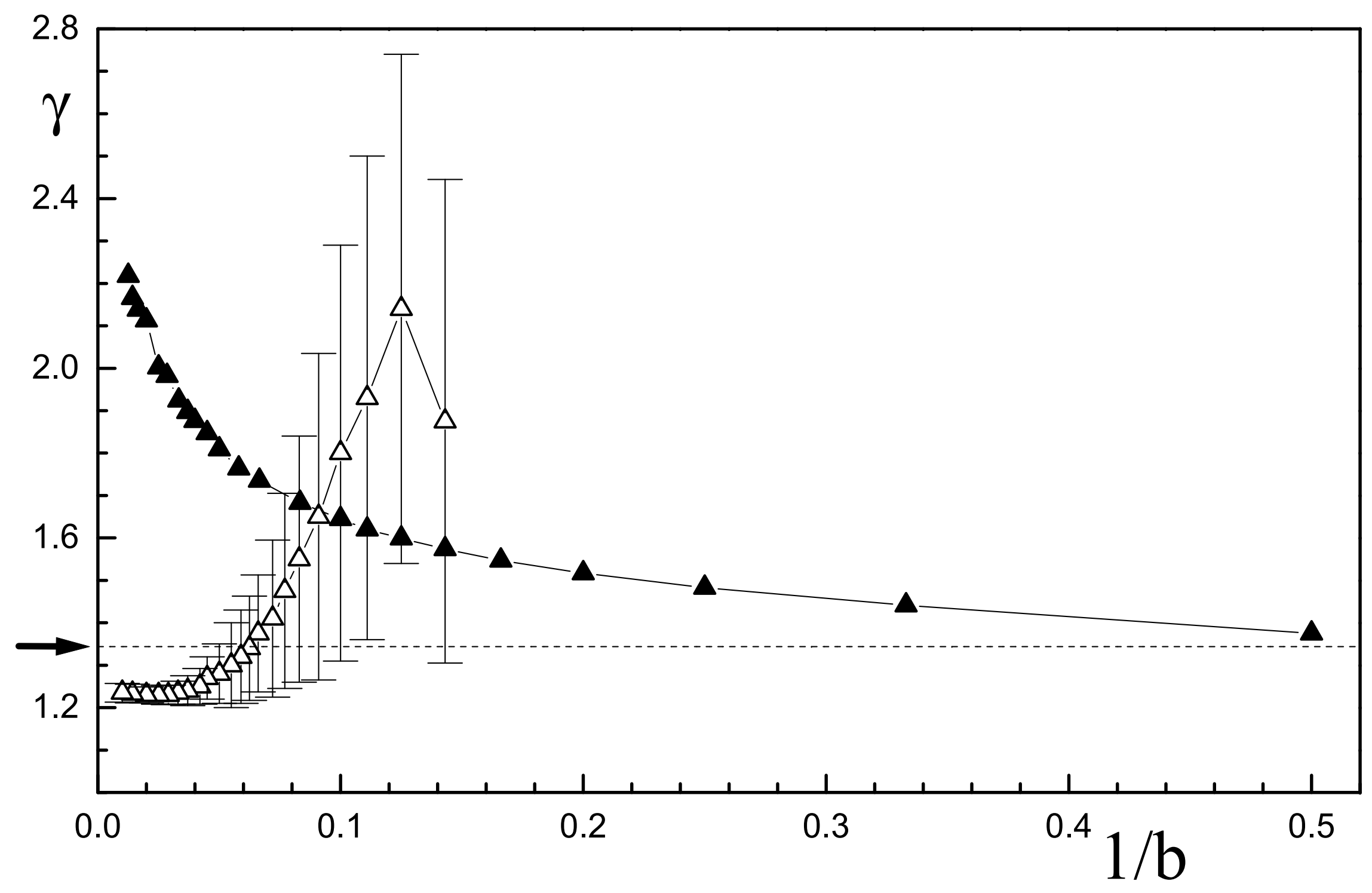




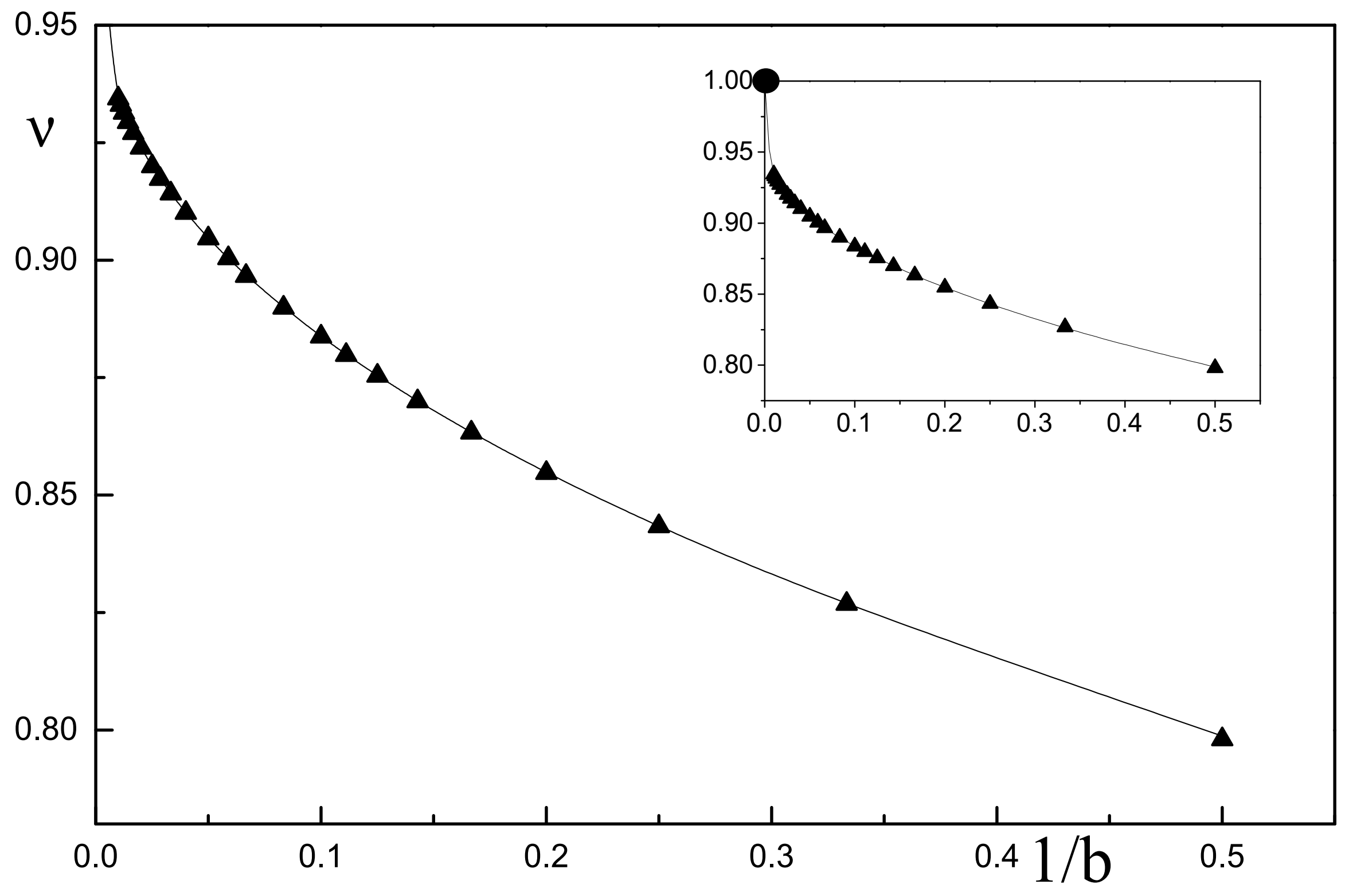




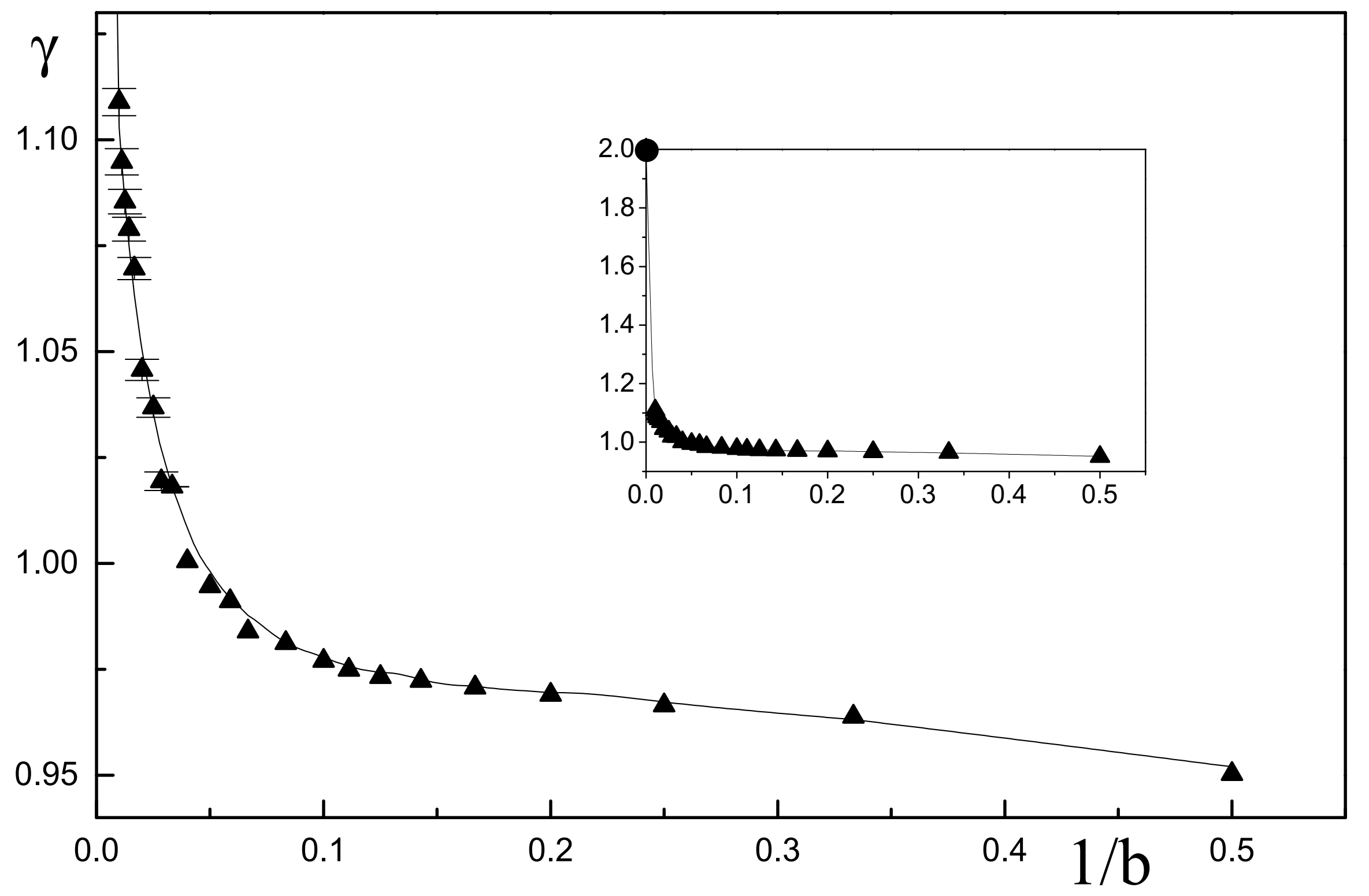




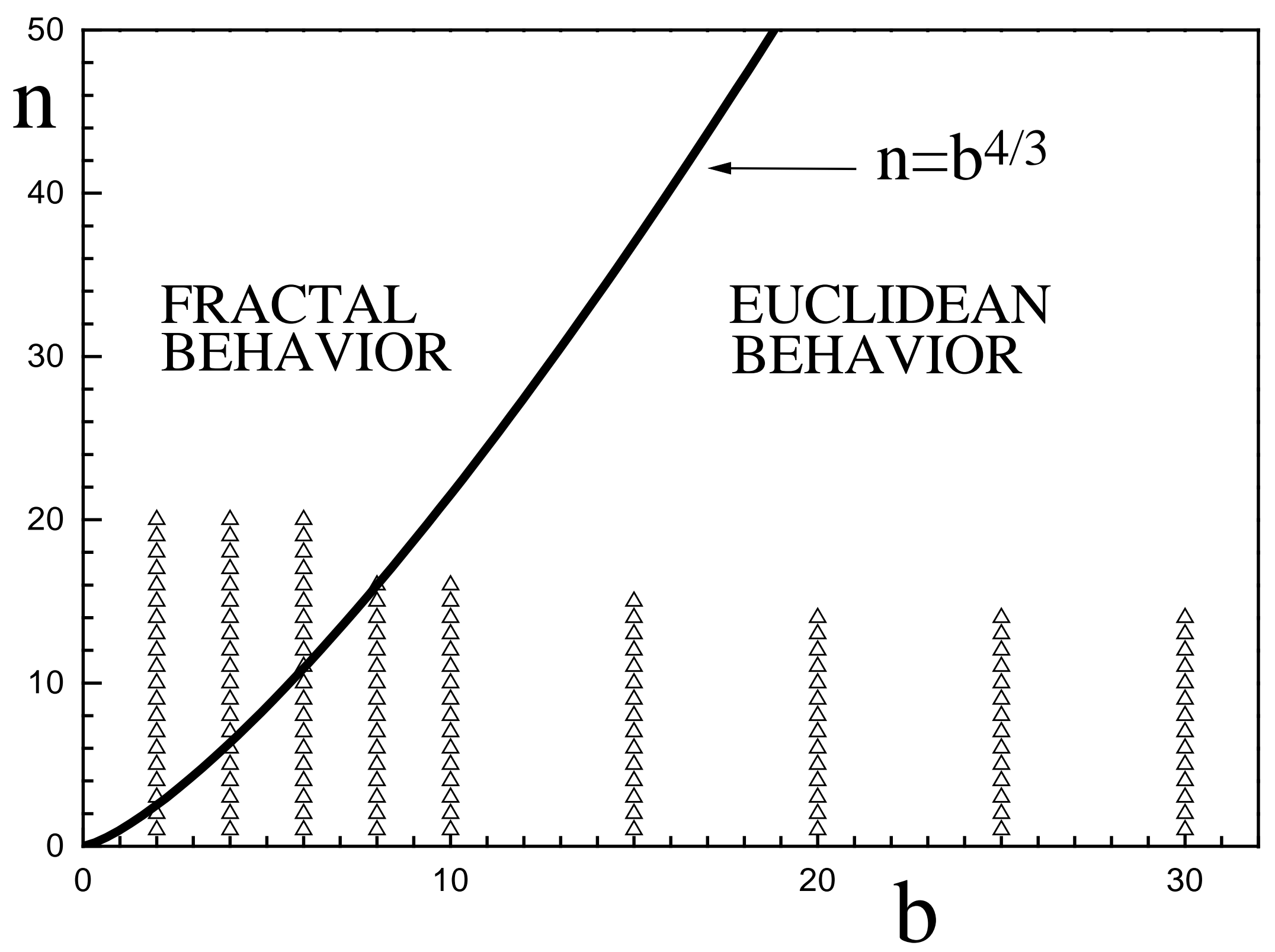

